Techniques \& Culture

Revue semestrielle d'anthropologie des techniques

$61 \mid 2013$

Vivre le sable !

\title{
La dune et la bosse
}

Dune and hump

\section{Bernard Faye}

\section{(2) OpenEdition}

Journals

Édition électronique

URL : https://journals.openedition.org/tc/7222

DOI : $10.4000 /$ tc. 7222

ISSN : 1952-420X

Éditeur

Éditions de l'EHESS

Édition imprimée

Date de publication : 15 décembre 2013

Pagination : 60-75

ISBN : 978-2-7351-1654-6

ISSN : 0248-6016

Référence électronique

Bernard Faye, "La dune et la bosse », Techniques \& Culture [En ligne], 61 | 2013, mis en ligne le 15 décembre 2016, consulté le 29 septembre 2022. URL : http://journals.openedition.org/tc/7222 ; DOI : https://doi.org/10.4000/tc.7222 


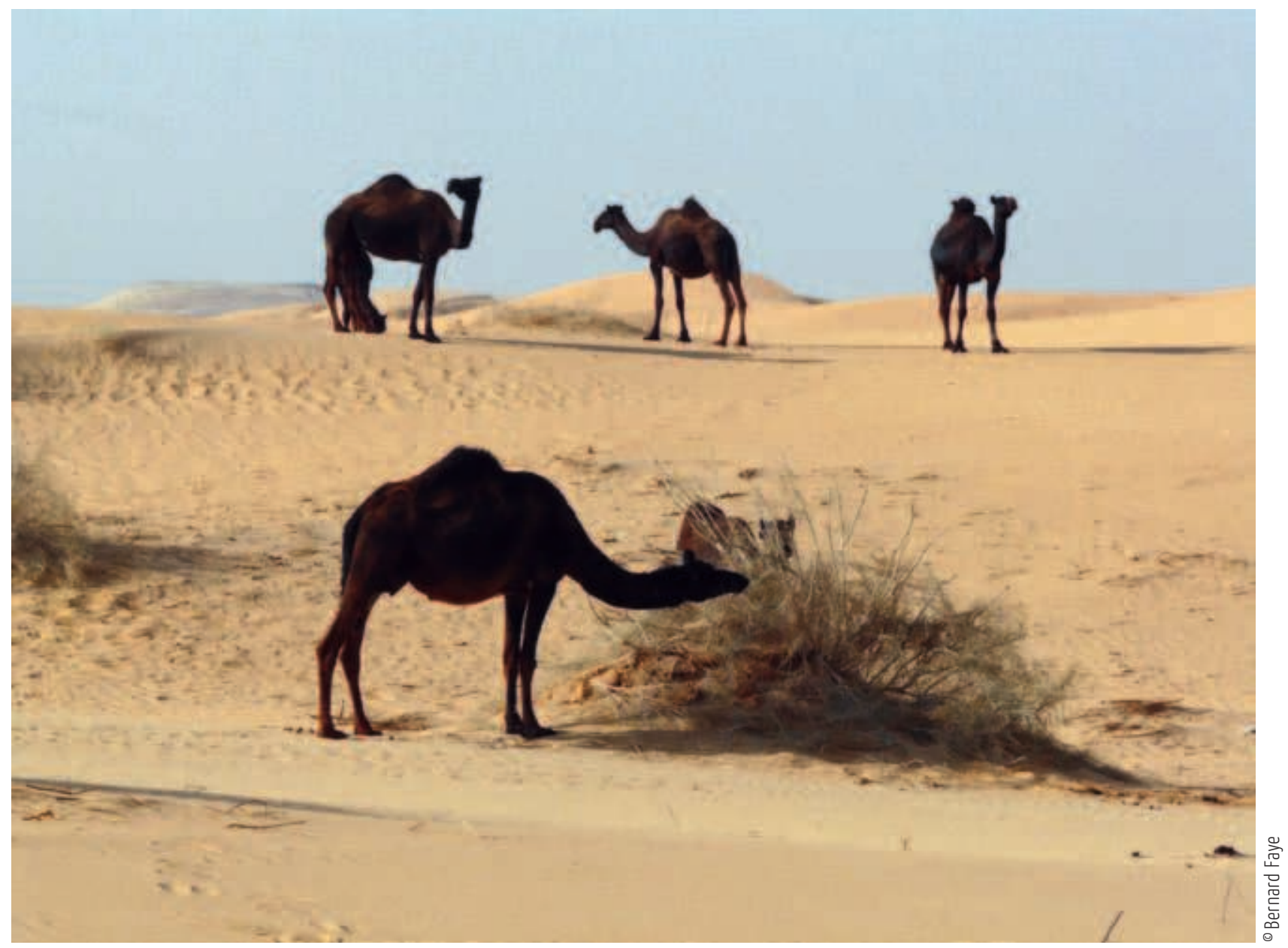




\section{LA DUNE ET LA BOSSE}

\section{Dromadaire : vaisseau du désert?}

Il y a des images d'Épinal qui ont la vie dure. Qui concevrait encore aujourd'hui le dromadaire sans paysage de dunes et un palmier dattier comme accessoire non obligé ? Ce chameau à une bosse ${ }^{1}$ qui hante nos rêves exotiques demeure l'emblème du Sahara, le plus grand des déserts de la planète, l'animal sans lequel l'homme ne saurait survivre en d'aussi hostiles contrées. Il reste le « vaisseau du désert », celui qui rend possible cette improbable traversée d'un monde dominé par le minéral, sable et pierre, sable surtout, s'arrêtant uniquement en ces îles que sont les points d'eau et allant d'un port-oasis à un autre, si possible vers ce rivage qui en arabe porte le nom de Sahel. Seul parmi les espèces domestiques, le dromadaire rend possible la confrontation des hommes à l'hostilité du désert, tout comme le renne l'autorise dans les contrées polaires ou le yak dans les altitudes glacées d'Asie Centrale.

Le dromadaire ne s'imagine qu'au travers d'antiques caravanes montant et descendant interminablement les dunes du Ténéré ou du Rub-Al-Khali, portant épices, blocs de sel et tomates séchées selon des pistes inamovibles que parcouraient déjà les explorateurs des siècles passés, de Zagora à Tombouctou, de Tamanrasset à Tahoua, d'Assouan à El-Obeid ou d'Aden à Jérusalem. Le balancement des bêtes assommées par le poids des marchandises et des hommes rythmerait-il encore, par cette marche à l'amble, ce qui reste de vie apparente dans un monde où la plus petite unité visible se réduit à un grain de sable ? Navire parfois en perdition, 


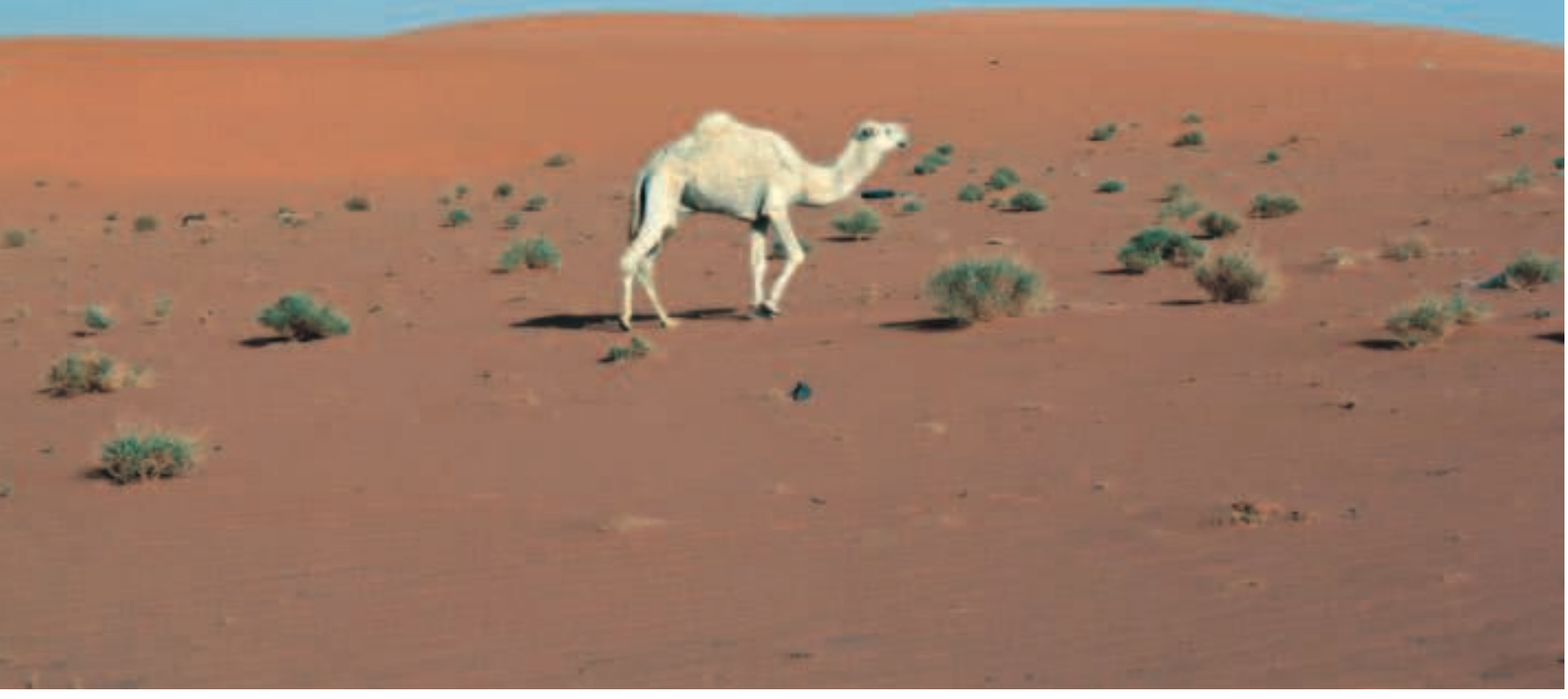

Dromadaires Waddah dans les dunes rouges du désert de Khowar en Arabie Saoudite

Le dromadaire est une des rares espèces d'herbivores capables de valoriser les pauvres ressources pastorales des zones sableuses

du désert. laissant son épave loin des points d'eau, carcasse aux os blanchis par le soleil, à la texture anatomique lissée par les vents de sable, et bientôt mangée par les dunes sans cesse en mouvement.

Et même si le dromadaire se mue en animal aux vertus zootechniques donnant son lait et sa viande dans de modernes unités de production, s'abritant sous d'authentiques bâtiments construits de matériaux nouveaux, se laissant traire par des machines ou des robots ou paradant dans les concours de beauté et les courses où se précipitent émirs et s'exprime ferveur populaire, il n'en reste pas moins l'animal du désert, attaché à ses dunes et aux rares touffes de graminées qui parsèment l'espace.

Et dans cette géographie dunaire se mariant parfois à l'âpreté des roches déchiquetées par une érosion chargée de grains de silice coupants comme des lames, la bosse du dromadaire n'est pas sans rappeler par son galbe unique dans le monde animal, la rondeur sensuelle des dunes du désert. La bosse et la dune de sable se répondent en miroir, monde animal face au monde minéral, tous deux façonnés par l'aridité, la permanence du vent et les écarts de température du jour et de la nuit (Monod 1992). L'un s'y adapte, l'autre en est l'ultime conséquence. Les écosystèmes les plus extrêmes façonnent-ils les espèces les plus adaptées à leur image? 


\section{L’anatomie de la sécheresse}

De fait, le dromadaire construit sa réputation proverbiale autour de son adaptation à tout ce qui caractérise le désert : extrême chaleur diurne, extrême sensation du froid nocturne, extrême sécheresse, extrême pauvreté des ressources alimentaires, extrême distance à parcourir pour accéder à l'eau, extrême violence des vents chargés de sable. Le dromadaire est l'animal des extrêmes. Parce que tout ce qui caractérise le milieu dans lequel il vit peut se résumer dans un grain de sable, brûlant le jour, glacial la nuit, sec au possible, infertile et assez léger pour cingler les cuirs dans les tourbillons du vent, et parce que l'animal a développé toutes les astuces anatomiques et physiologiques pour résister à un tel acharnement de la nature, il y a entre le dromadaire et le sable comme une entité contradictoire associant le biologique et le minéral pour que l'un annihile les effets de l'autre sans quoi la survie animale ne serait plus possible, sauf à faire comme les petites espèces qui hantent les ergs et les regs, lézards, serpents, gerboises et fennecs, à vivre terré au plus profond du sol, s'éloignant de la chaleur et de l'aridité. Le dromadaire, lui, compte tenu de sa taille n'avait pas le choix. Il se devait d'affronter le désert et ses grains de sable sans se dérober.

L'eau se perd dans les sables et les gouttes de rosée s'évaporent aussi vite qu'elles tombent des rares feuilles sèches des xérophytes. Le dromadaire, au contraire, économise tout ce qu'il peut de l'eau qu'en de rares occasions il a pu ingurgiter en grandes quantités. L'eau ne se perd pas dans les entrailles de l'animal. Elle est stockée dans les estomacs, dans le sang, dans tous les espaces intercellulaires de l'organisme. Ses globules rouges agissent comme des éponges, se gorgeant d'eau tout en résistant à la forte pression osmotique que

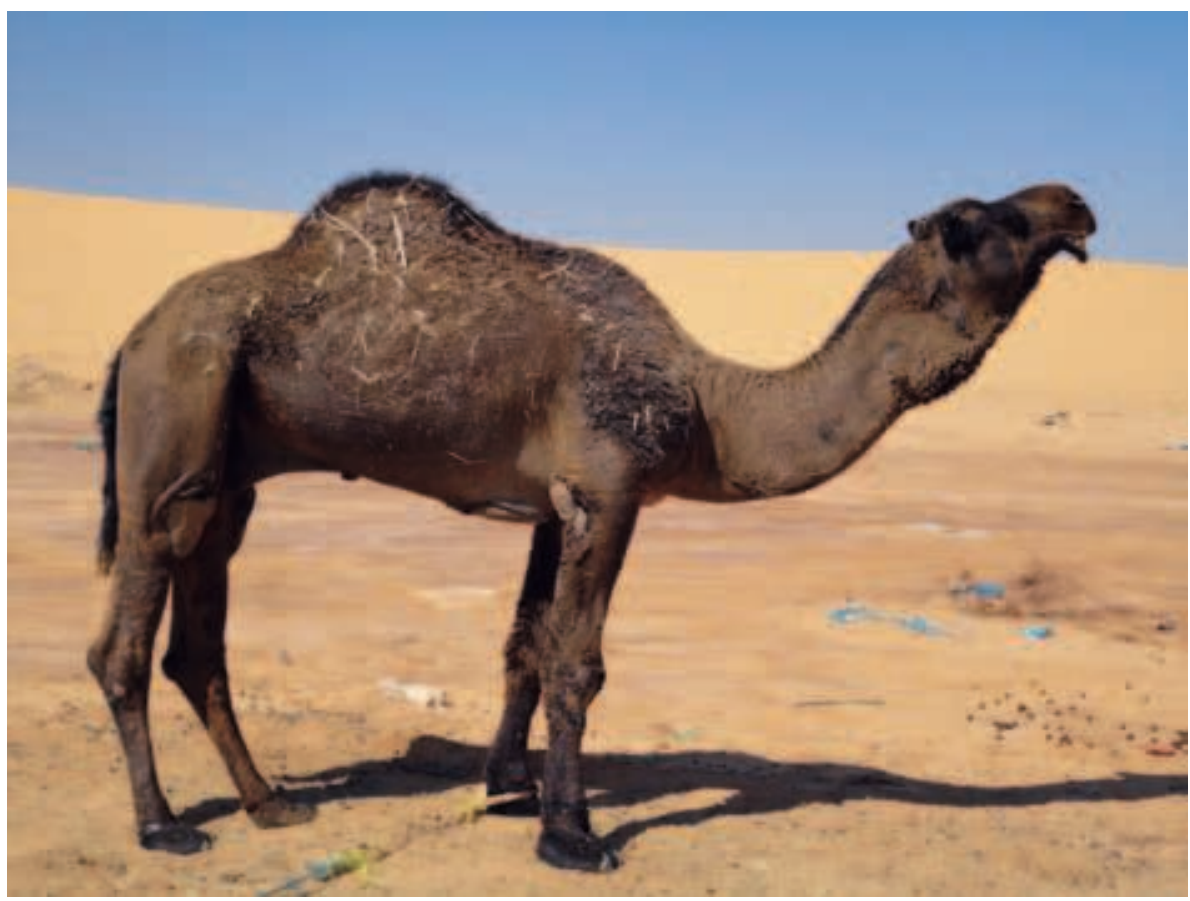

Dromadaire Majaheem avec sa bosse bien arrondie dans les dunes du désert de Nafud en Arabie Saoudite, bosse et dune marquées par l'homologie de leur rondeur. 


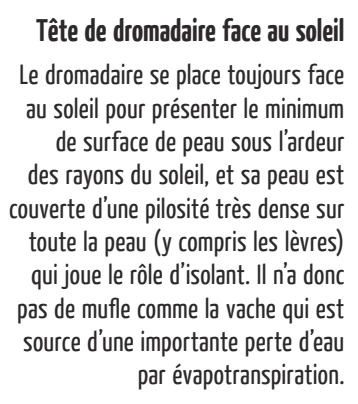

Tête de dromadaire face au soleil

Le dromadaire se place toujours face au soleil pour présenter le minimum de surface de peau sous l'ardeur des rayons du soleil, et sa peau est courte d'une pilosité très dense sur pas de mufle comme la vache qui est par évapotranspiration.

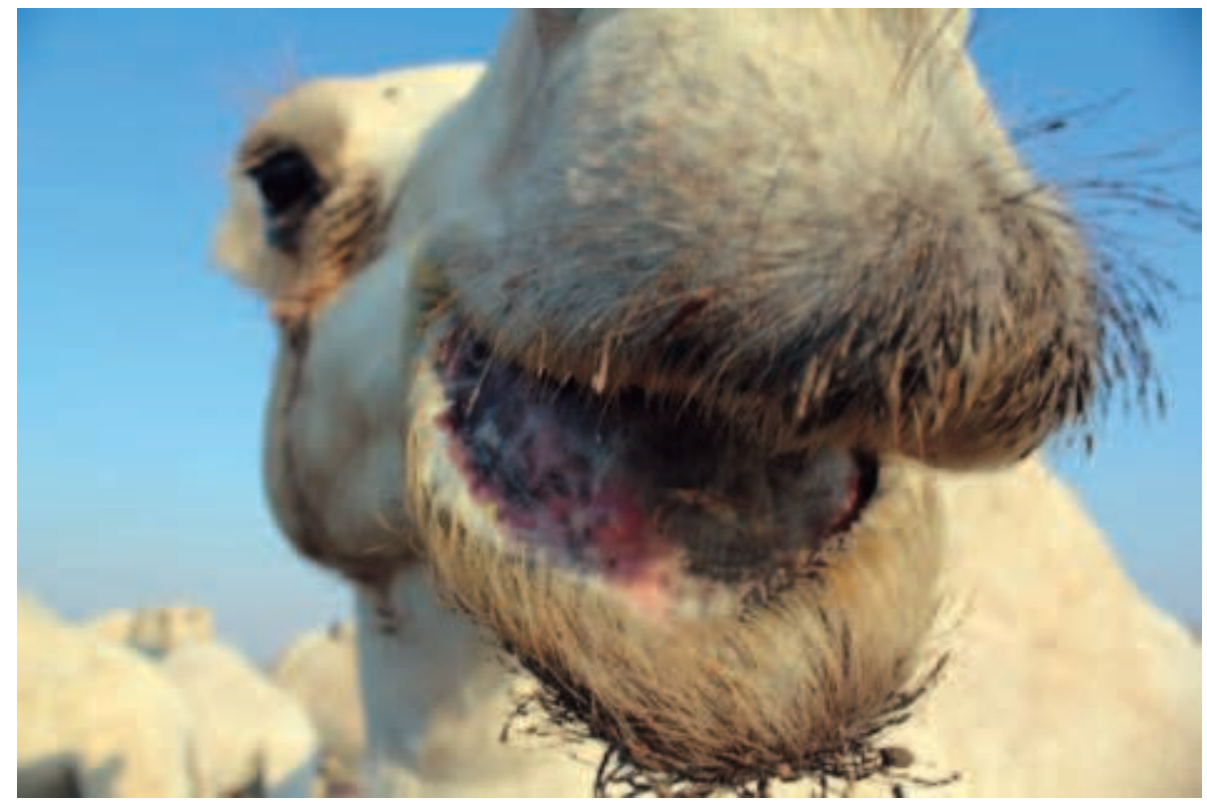

cet apport soudain d'eau induit. Toute autre espèce engagée dans le même processus de déshydratation/réhydratation en mourrait. Et toute cette eau, contrairement à celle que reçoit le sable, ne s'évapore pas aussi vite. Condensée dans les circonvolutions sinusales lors de l'expiration, récupérée dans les reins lors de l'excrétion urinaire, réutilisée jusque dans les anses intestinales lors de l'émission des crottes tellement sèches qu'elles font un bruit mat en tombant sur le sable. Minimum de sueur, urine concentrée, crottes de plomb. Le dromadaire rend au sable le minimum d'humidité. Il tourne à l'économie. Il conserve en lui ce que le sable est incapable de garder (Bengoumi et Faye 2002).

Le sable brûle le jour, glace la nuit. Il ne tempère rien. Il avale la chaleur du soleil. Il absorbe le froid de la lune. Pendant ce temps-là, notre animal module les extrêmes. Son poil dense et court crée un microclimat autour du corps, protégeant du froid pendant la nuit, brisant la chaleur du jour. Le sable brûle, étouffant l'air à proximité? Ses membres démesurément longs aident à maintenir la circulation d'air autour des organes vitaux de l'abdomen. Sa température interne augmente de quelques degrés, histoire de diminuer les écarts de température avec l'extérieur et d'économiser l'eau. Il se met face au soleil, présentant le minimum de surface de peau aux ardeurs de ses rayons. Le sable gèle après le crépuscule? Sa température diminue de concert, son métabolisme se ralentit, son thermostat interne fait ce que le sable ne peut pas : il atténue les effets délétères du trop chaud et du trop froid (Faye 1997).

Le sable est stérile. Seules quelques plantes courageuses et dispersées y plongent des racines profondes pour chercher un minimum d'humidité loin de la surface, des plantes épineuses, aux feuilles réduites à leur plus simple expression, souvent de faible valeur nutritive. Qu'importe, le dromadaire s'en contente, tant il est capable de récupérer au niveau rénal et intestinal, la plus grande partie des nutriments essentiels à sa survie, azote, eau, minéraux, avant même qu'ils ne soient exportés dans l'urine et les crottes comme cela survient chez la plupart des autres animaux domestiques (Yagil, 1985). Et si les plantes sont disséminées sur un vaste espace, notre animal saura grappiller sur de 
longues distances, les quelques touffes nécessaires à assurer son embonpoint concentré dans la bosse. Recyclage des nutriments, limitation de l'excrétion, pâturage ambulatoire, sélection des meilleures plantes, diversité des espèces consommées : le dromadaire sort toute sa panoplie physiologique et comportementale pour compenser la stérilité apparente du désert de sable. Il rend productif ce qui n'est qu'en apparence survie.

\section{Les pieds dans le sable, la tête face au vent}

Le sable est meuble comme le dromadaire est mobile. Il glisse sous les pas, rendant toute marche épuisante, et les traces que l'animal et son chamelier laissent sur les dunes n'ont que la permanence que veut bien leur laisser le vent, ce vent du désert qui déplace les grains de sable en brûlant les yeux, en pénétrant dans les narines et la bouche, ce pourquoi les nomades doivent s'en protéger en s'enveloppant dans leur chèche. Pourtant, le dromadaire navigue sur la mer de sable, harcelé par les salves de silice sans paraître s'en soucier. C'est qu'il a le pied marin et la tête hermétique.

Le pied est large, souple et robuste. Une anatomie particulière, un ensemble comblé de tissus conjonctifs d'une élasticité remarquable avec entre le réseau des ligaments assurant la flexibilité de l'organe, deux petites boules de la taille d'un œuf de poule, d'une texture unique dans le monde animal, à la fois solide et malléable. Elles donnent au pied la capacité d'épouser les accidents du terrain sans en souffrir. Le tout recouvert d'un épiderme corné protégeant de la brûlure du sable. Et qui donne un pas non traumatisant pour le sol. Un anatomiste facétieux s'amusait à dire dans les conférences

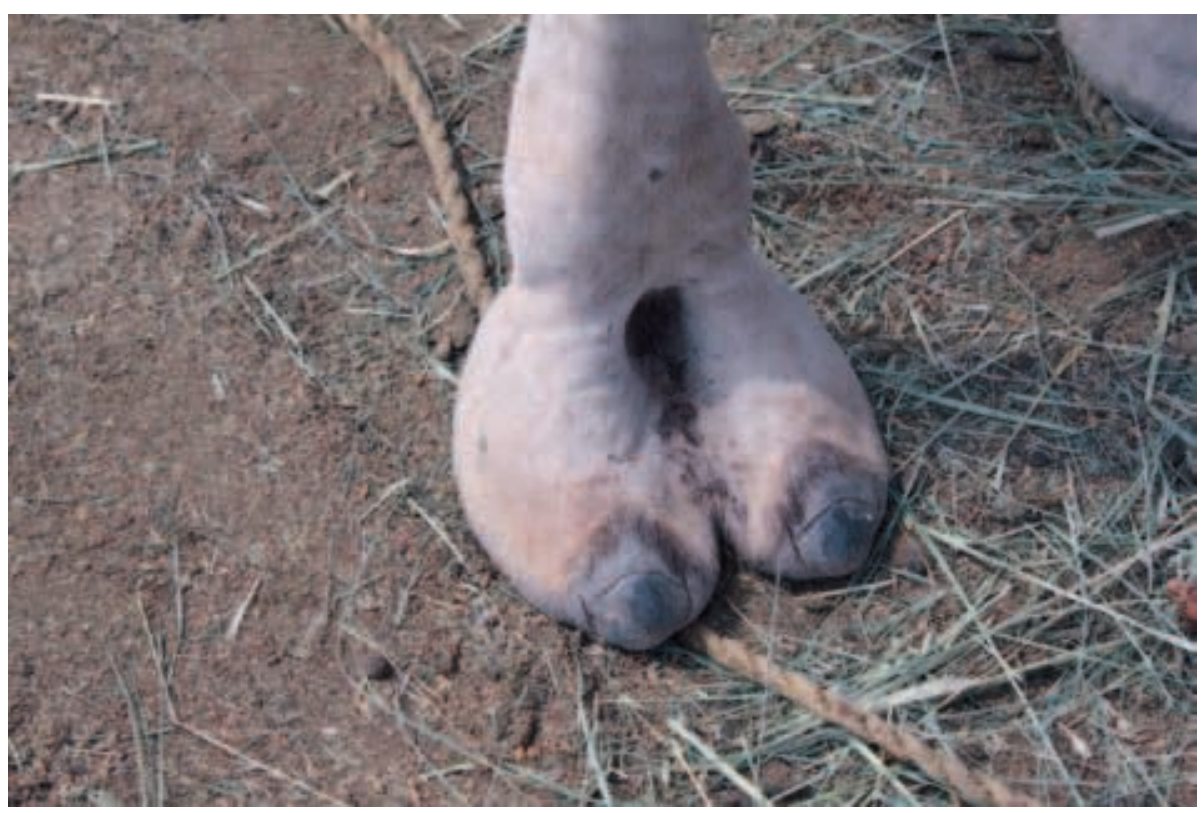

\section{Pied de dromadaire}

Le pied du dromadaire est remarquable par sa souplesse et sa largeur, sa capacité à s'adapter à l'irrégularité du terrain et à supporter les écarts de températures du sable entre le jour et la nuit. 
Profil d'une tête de dromadaire remarquable par les longs cils protecteurs du vent de sable, des narines fermées et un port de tête horizonta limitant la prise au vent.

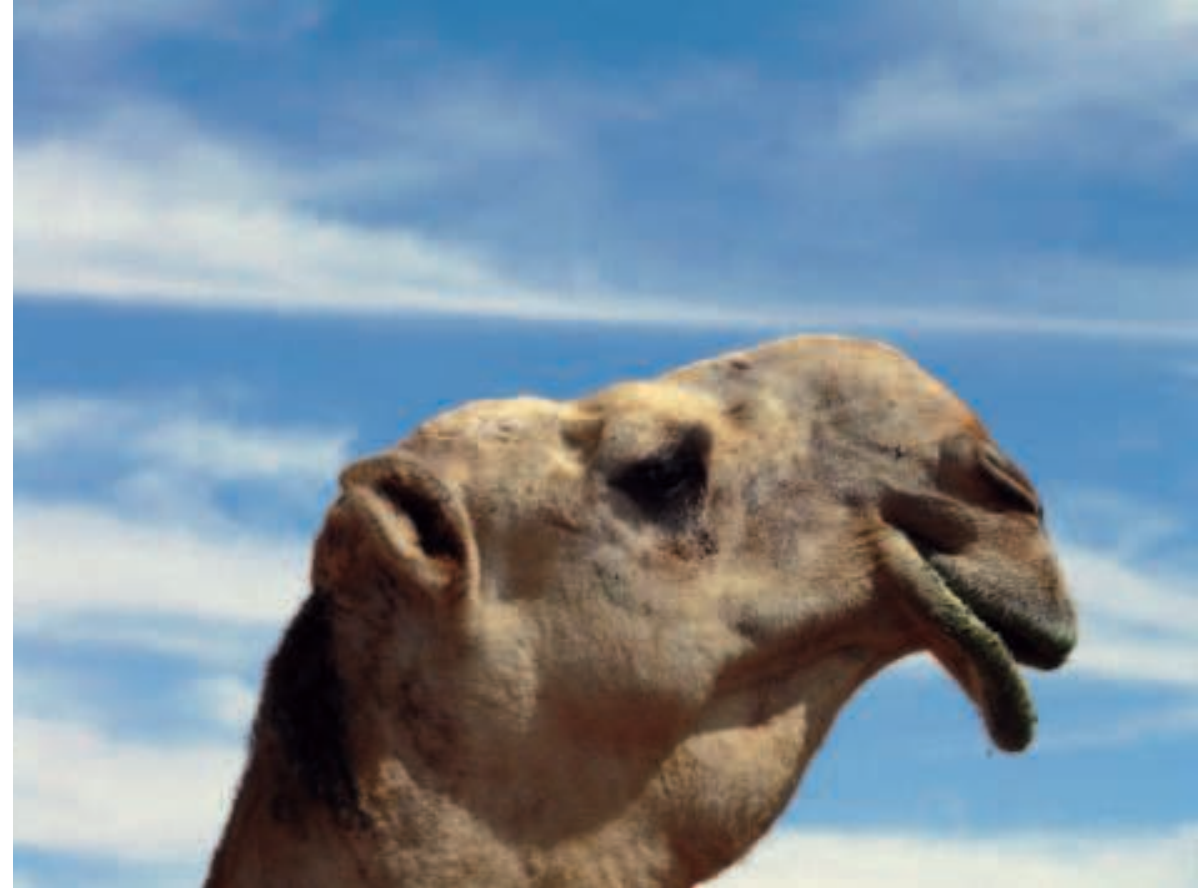

consacrées aux camélidés, que notre animal était plus moderne que la vache ou le cheval : alors que ceux-ci marchaient encore en sabots, le dromadaire avait depuis longtemps opté pour des chaussures sportives qui avaient fait le succès d'une célèbre marque américaine (Arnautovic 1997). Une merveille anatomique qui s'adapte parfaitement à la fugacité du sable.

Et que dire de la tête, portée haute, donnant à l'animal cet air de dédain qui lui confère l'apparence d'être sur un piédestal. Des yeux aux longs cils pour protéger le globe oculaire des fureurs du vent chargé des grains de silice. Des narines qui se ferment comme des ventaux en cas de tempête. Un port de tête horizontal minimisant la prise au vent. Et une langue étroite et souple qui lui permet d'accéder aux parties les plus protégées de la contamination par le sable au sein des touffes de plantes, évitant ainsi l'usure prématurée des dents, sans quoi son espérance de vie serait raccourcie. Et limitant aussi l'ingestion trop abondante de ce sable qui déprime la digestibilité chez les espèces comme la vache et le mouton, animaux moins sélectifs que le dromadaire et habitués à brouter les herbes au plus près du sol. À tout prendre, le dromadaire s'accommode plus aisément de l'omniprésence du sable que tous les autres brouteurs de la planète.

\section{Des crottes dans le sable}

Que reste-t-il du passage des troupeaux sur le sable quand le vent en a effacé les traces? Rien, sinon des petites boulettes brunes et sèches. Des crottes! Avec le temps, la boule 
se délite et retourne à la poussière tellement le sable ne sait retenir le peu de fertilisants que les excréments des herbivores sont censés contenir. Aucune valorisation possible aussi longtemps que les hommes ne s'évertuent pas à ramasser cette matière pour la revendre aux maraîchers des bords de ville, comme cela se pratique au Rajasthan. Mais dans le désert de sable, dans cette immensité minérale où la mobilité est permanente, qui va s'échiner à collecter cette manne ? La crotte du dromadaire n'est pourtant pas qu’un détritus inutile. À bien des égards, elle est indispensable. Aussi étonnant que cela puisse paraître, la crotte représente un enjeu écologique, un enjeu scientifique et même un enjeu social.

Écologique? Les graines des plantes du désert sont comme le sable. Dures, coriaces, en apparence indestructibles. Mais contrairement au sable, insensible aux assauts des sucs digestifs, les graines ingérées par le dromadaire se retrouvent dans l'intestin, bien enrobées par la matière fécale riche en cellulose, leur tégument amolli, moins ferme et donc moins résistant à la pression germinative de la plantule en son sein. Une fois à l'extérieur, le sable étant d'une neutralité avérée pour la survie des plantes, la petite graine ne pourra germer que grâce à l'environnement favorable de la crotte, en attendant que les racines puisent les éléments nécessaires à leur survie dans les profondeurs. Ainsi, le dromadaire aide à la dispersion des rares ressources du désert, aide à conquérir un minimum d'espace vital dans les univers désertiques. Dans le Sahara septentrional algérien, 35 graines appartenant à autant d'espèces différentes ont ainsi été identifiées dans les excrétions de notre animal à bosse (Trabelsi et al. 2012). Il est ainsi celui par lequel le sable ne retourne pas à son éternelle stérilité. Il est celui par lequel le désert reste habitable maintenant d'héroïques buissons qui s'adaptent à l'omniprésence du sable.

Scientifique? La crotte est un fantastique résumé de ce que les ressources pastorales de la nature ont offert à l'animal qui s'en nourrit. Difficile de suivre le dromadaire dans ses pérégrinations entre touffes de Panicum, de Cenchrus ou de Stipagrostis; de savoir exactement ce qu'il ingère au cours de ses déambulations. Par une analyse morphologique des parois cellulaires retrouvées partiellement intactes dans la crotte, le chercheur peut reconstituer la ration alimentaire de notre animal, les plantes qu'il a ingérées et donc le type de pâturage par où il est passé (Correra et al. 2011). Et ainsi retrouver les traces d'un passage que le vent de sable croyait avoir fait disparaître.

Social ? Toute l'Afrique connaît ce jeu consistant à compter et capturer des cailloux, des graines ou des coquillages dans des coupelles ou des trous, parfois creusés à même le sol. Un jeu de stratégie combinatoire très populaire qu'on désigne sous le nom générique de mancala, mais qui peut porter plusieurs autres noms comme awalé ou bao ou nam-nam. Dans les sables du désert, les coupelles ne sont que des godets à peine creusés et les pions des crottes de dromadaire bien séchées au soleil. Des pions que l'on peut peindre. Dès lors le sable et la crotte du dromadaire s'associent pour un instant afin de satisfaire l'universel goût ludique des hommes.

\section{Traces de dromadaires dans le sable après le passage d'un troupeau}

Les bons éleveurs sont capables de repérer leurs animaux par la lecture des traces laissées sur le sable.

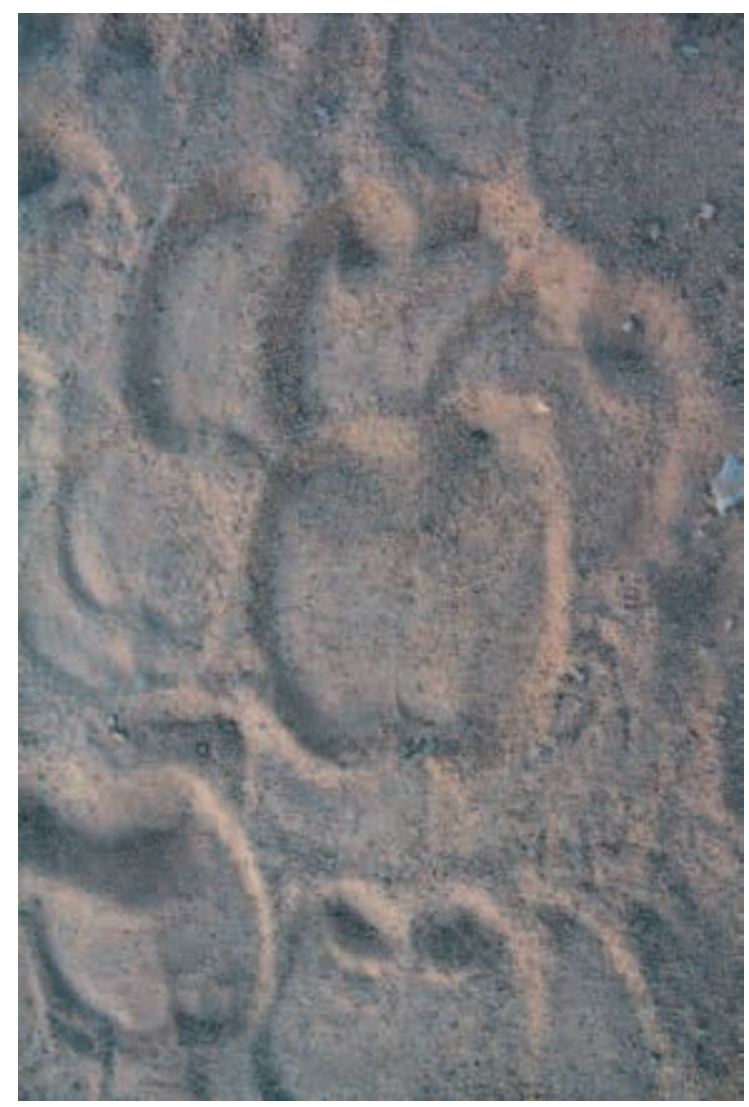




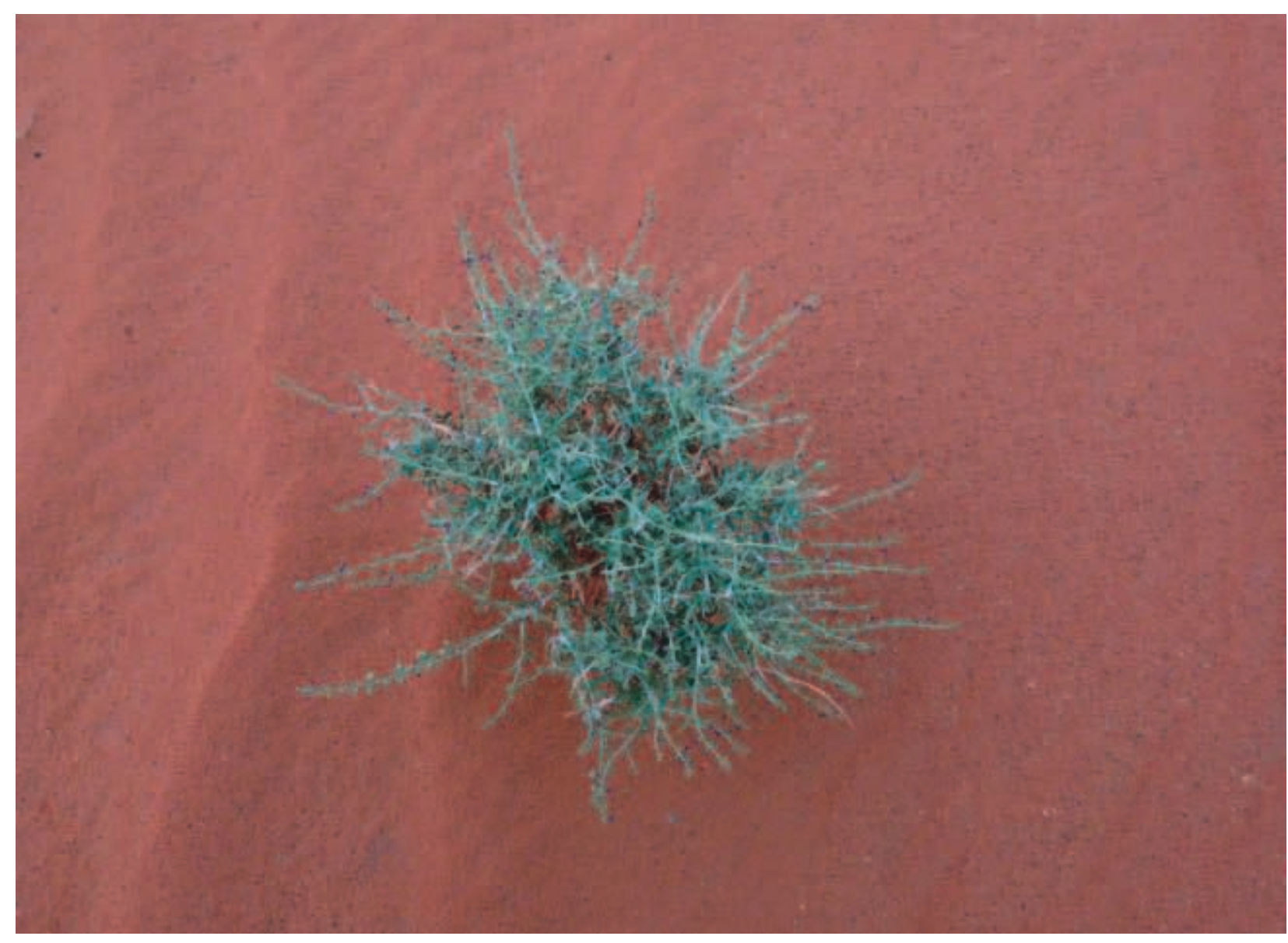

\section{Plante xérophyte (Traganum nudatum) du désert}

Pour germer dans un milieu auss stérile que le sable, les plantes émettent des graines dont le pouvoir germinatif augmente après leur transit dans le tractus digestif du dromadaire.

\section{Le sable, un lieu de passage}

L'erg, ce lieu où le sable règne en maître au cœur des déserts, est un espace aux allures d'infini. Certes, il n'est pas le seul sur notre planète à pouvoir revendiquer une telle sensation d'immensité. Tout comme l'erg, la haute mer, les plateaux glacés des pôles, les successions de pics enneigés sont des lieux rappelant à l'homme sa petitesse et sa fragilité. Et tout comme ceux-ci, l'erg n'est qu'un lieu de passage. Des espaces parcourus par d'intrépides marins, explorateurs ou alpinistes. Mais ce ne sont pas des lieux de vie pour l'homme. Dans le sable des dunes, seuls passent de rares caravanes ou des troupeaux de dromadaires allant d'un lit d'oued à l'autre entre deux cordons dunaires, et les rares habitats ne sont que des bivouacs de fortune. Les lieux de vie, eux, se rencontrent dans les espaces plus accueillants des ouadis où l'eau parfois affleure au creux de quelques rochers bienveillants. Qui n'a pas été envoûté par la magie des gueltas ${ }^{2}$ du Sahara ? Les hommes du désert cherchent l'ombre bienfaisante des arbres, fussent-ils des épineux. Ou bien celle rafraîchissante des palmiers dattiers aux alentours des oasis. 
L'homme du désert ne se satisfait du sable que parce qu'il dessine la courbe des pistes caravanières. On traverse ces espaces. On ne s'y installe jamais. Quand dans le passé, des hommes ont construit d'austères bâtisses de pierre ou de terre en des lieux reculés du désert, l'ensablement des maisons et des puits marquait la fuite des habitants. De loin en loin, la désertification s'accompagne ainsi de la désertion. Et ces villages fantômes qui hantent parfois les historiques pistes caravanières ne deviennent plus que les lieux d'un campement éphémère. Le sable est l'ennemi de la sédentarité. Il est le moteur de la mobilité des hommes et de leurs troupeaux de dromadaires. On ne s'étonnera donc pas de s'apercevoir que l'intensification de l'élevage de nos animaux à bosse donne sens à des systèmes de production marqués du sceau de la sédentarité loin des dunes de sable, aux marges du désert, en des lieux où l'eau permet d'avoir des cultures fourragères irriguées, des infrastructures routières et architecturales à l'abri de l'ensablement, et des lieux de vie appréciés des hommes. Quand on survole le nord de l'Arabie Saoudite, on ne peut être que frappé par cette immensité vide du désert du Nafoud entouré en ses marges par d'immenses cercles verts, fruits de l'irrigation sur pivot des champs de luzerne destinés à l'alimentation d'animaux désormais voués à la sédentarité forcée.

\section{Éleveurs chameliers, entre sable et sédentarisation}

En effet, si le sable pousse à la mobilité des troupeaux accompagnés des bergers, le nomadisme est en conséquence la quintessence de l'élevage camelin. La mobilité implique le savoir des chemins et de la richesse cachée dans les dunes de sable et les déserts de pierre. De fait, l'homme conduisant son troupeau de dromadaires parmi les dunes sait mieux que quiconque le secret de la productivité du désert. Il n'est aucun berger du Sahara ou du Moyoum-Koum, du Nafoud ou du Gobi qui ignore le nom et les propriétés de chacune des plantes dont raffolent les troupeaux. Ils n'en connaissent pas la valeur alimentaire calculée en énergie brute ou en taux de protéines, mais ils savent le pouvoir sur la production de lait ou sur la croissance des jeunes. Ils disent, celle-là fait arrondir la bosse, celle-ci donne un bon goût au lait, et celle-là, encore plus loin, est à éviter pour les jeunes mais elle donne de la vigueur aux mâles du troupeau. Même rabougries, les feuilles pulvérisées de trop de sécheresse, le berger sait reconnaître M'Rokba (Panicum turgidum) de Sardoun (Stipagrostis acutiflora) ou bien sbot (Stipagrostis pungens) de Nsil (Stipagrostis ciliata). Certes, la divagation (le hmil de la steppe algérienne) est quelquefois le mode en usage dans les zones désertiques, considérant que le dromadaire est fort bien capable de trouver ce dont il a besoin sans le recours d'un gardien (Chehma et al. 2008). Mais dans la plupart des cas, le chamelier possède la science des bons pâturages, fussent-ils clairsemés au pied des cordons dunaires. Il saura dès lors guider le troupeau, là où les touffes de graminées sont encore mangeables, ou bien, miracle, en un lieu où trois gouttes de pluie soudaine, ont fait exploser une annuelle que nul n'avait vue depuis des années, une léontice, un asphodèle ou un héliotrope aussi soudainement abondant qu'éphémère.

Loin du sable, aux confins des dunes, le chamelier sédentarisé perd la connaissance du monde végétal qui l'entoure. Et la ration alimentaire du chameau désormais 
« civilisé » devient monotone. Habitué à grappiller les cent espèces différentes qui hantent les sols sableux et les lits d'oued du lever au coucher du soleil, voilà notre dromadaire s'obligeant à deux repas par jour, limités à trois ou quatre ingrédients, du foin issu d'une culture fourragère irriguée, un peu d'orge pour l'énergie, parfois un concentré du commerce pour le lait, plus rarement un complément minéral. Le passage du sable à l'humus s'accompagne de l'oubli des richesses du désert (Rutagwenda et al. 1990).

Désert de sable, donc nomadisme. Nomadisme, donc élevage traditionnel. Élevage traditionnel, donc faibles performances. Un raccourci commode. On en oublierait que la tradition est aussi un savoir. Que le nomadisme est une stratégie. Une stratégie de survie, de sécurisation du système de production, de valorisation optimale du milieu. Dans ces conditions, le but n'est pas de produire plus, ni de vendre à tout prix. Il suffit de conserver l'équilibre. Suffisamment d'animaux pour reconstituer le cheptel en cas de surmortalité, n'assurer la reproduction que si l'on est certain de pouvoir satisfaire aux besoins du jeune qui va naître, espacer les naissances pour coller aux saisons favorables, gérer la ressource pastorale en équilibre avec la ressource en eau, n'acheter des fourrages qu'en cas de « soudure » difficile, vendre quand il est besoin d'avoir de la trésorerie. C'est ainsi que se maintient l'élevage camelin traditionnel nomade en milieu désertique. Il reste cependant des bédouins qui s'enfoncent encore dans le désert en cédant parfois à la modernité pour se soustraire à la contrainte du point d'eau en mobilisant la ressource hydrique dans les camions-citernes, qui stockent un peu de fourrages ou d'herbes de brousse en complément, transportés à l'arrière de leur " Toyota pick-up ». Dès lors, les troupeaux ne vont plus à l'eau et à l'herbe mais l'eau et l'herbe vont aux troupeaux. Et ceux-ci valorisent encore mieux les ressources les plus marginales.

Mais il faut pourtant au chamelier quitter ce désert de sable, enclore ses bêtes, les nourrir « hors-sol » pour rejoindre la cohorte des « élevages modernes». Le lait, ce don de Dieu qui s'offre à l'hôte de passage que tout nomade aime à honorer, se vend désormais à prix d'or dans les supermarchés. La machine à traire fait son apparition et les marchands d'aliments pour chameaux vont d'une ferme caméline à l'autre, car le chamelier a cessé de vivre dans un «campement » aussi mobile que le dromadaire. Celui-ci ne

\section{Puits ensablé dans le désert \\ de Mauritanie \\ marquant l'aridification du milieu}

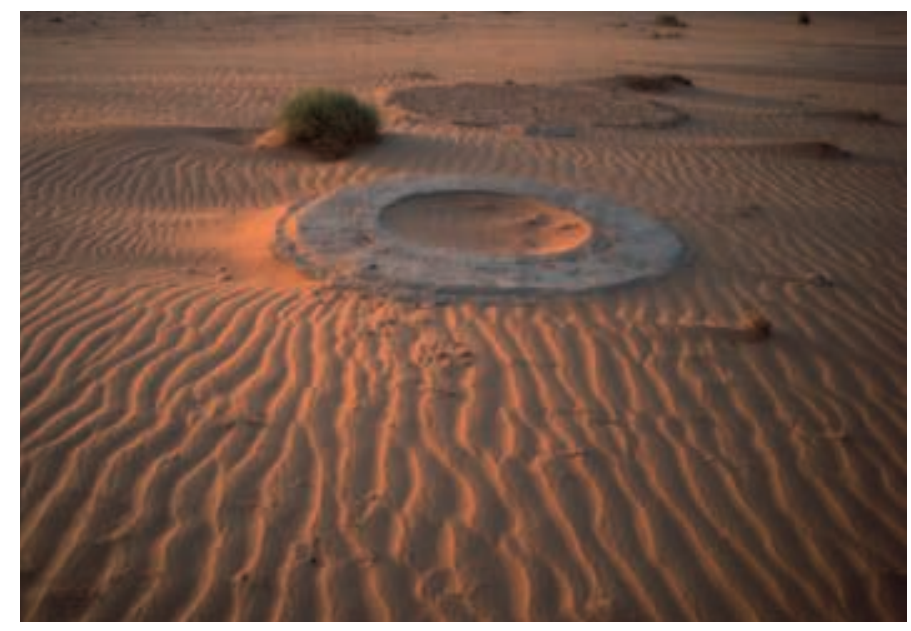




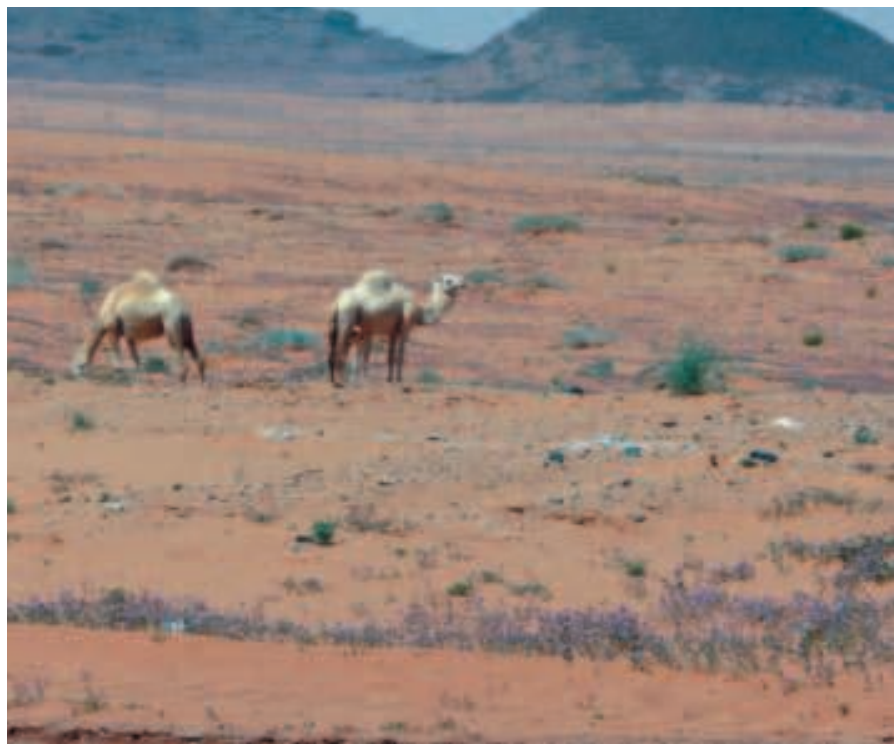

porte plus la maison des nomades sur son dos. Il devient une machine à fabriquer du lait. Ou de la viande. On « biotechnologise » sa reproduction dans les instituts de recherche en s'essayant à l'insémination artificielle et au transfert d'embryon. On rationalise son alimentation désormais monotone. À l'extrême, surgissent par exemple dans les pays du Golfe d'immenses fermes de plusieurs milliers de têtes, avec leurs parcs séparant les animaux selon leur statut (en lactation, gestantes, jeunes, reproducteurs), leur distributeur automatique de concentrés, leur robot de traite, leur unité de pasteurisation du lait associée, leur hangar de stockage des fourrages et leurs cultures fourragères irriguées. Le désert et son sable ne sont pourtant pas loin. Il suffit d'aller au-delà des cercles de cultures irriguées pour retrouver les dunes désormais désertées. Le désert de sable s'accommode mal de la modernité en élevage (Faye et al 2002).

\section{Restera-t-il des déserts de sable pour l'élevage du dromadaire?}

Si la tendance s’accélère, que restera-t-il du cour du désert ? Il ne maintenait un minimum de sentiment vital que grâce à la pugnacité du dromadaire et des chameliers, faisant des dunes de sable un lieu de passage dans lequel se dispersaient les graines, et poussaient quelques touffes de plantes ingrates dont se satisfaisaient les animaux. Si le dromadaire abandonne le sable pour les parcs à bétail des terres irriguées, deviendra-t-il ce citadin obèse qui ne saurait retourner dans les profondeurs du désert, loin des lumières de la ville? Perdra-t-il ces capacités d'adaptation qui durant des millénaires ont fait sa force et assuré sa survie? Le dromadaire s'adaptera-t-il s'il quitte son sable? Son lait et sa viande auront-ils toujours les mêmes vertus qu'on leur prête quand la ration peu variée qu'on lui donnera naura plus rien à voir avec la diversité des plantes du désert? Que deviendra sa bosse sans la dune? 

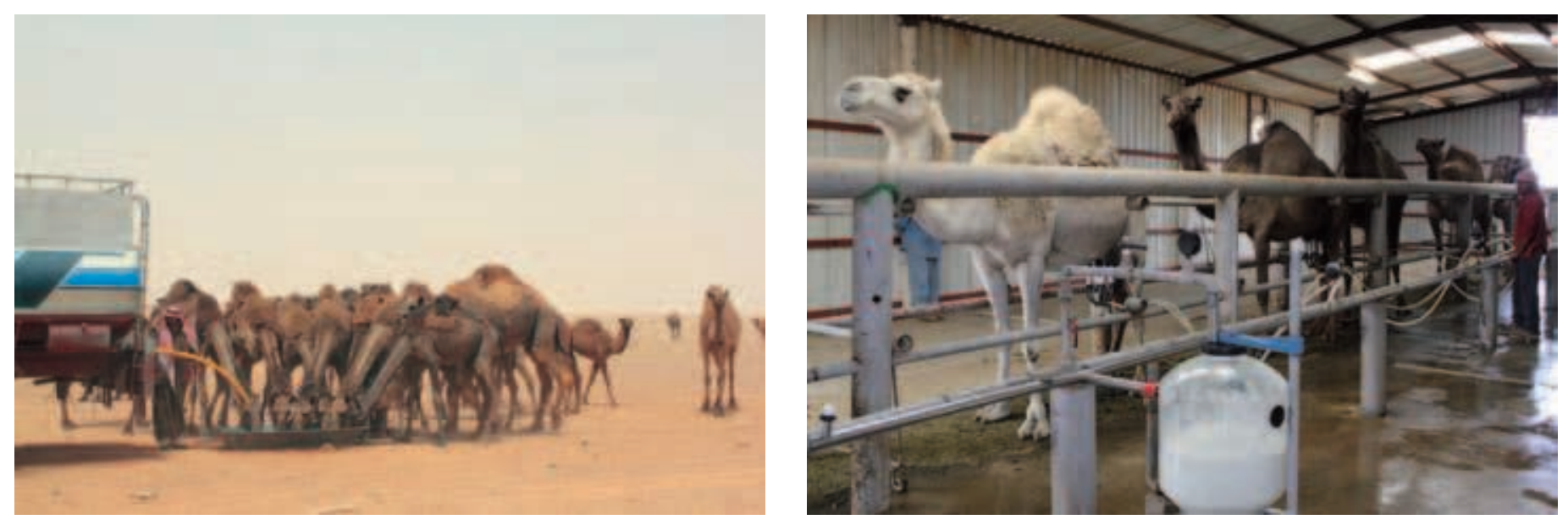

\title{
Dromadaires
}

dans une salle de traite moderne (ferme Watania en Arabie Saoudite)

Le dromadaire en élevage intensif entre dans une certaine « modernité », mais avec quelles conséquences sur la qualité des produits?

\begin{abstract}
$\&$
La force de l'homme est d'avoir su s'adapter au monde. En tout temps. En tout lieu. Le chamelier ne fait pas exception dans les sables du désert. En Mauritanie, se sédentarisent les troupeaux de chamelles laitières autour des grandes villes pour donner leur lait aux laiteries de la modernité industrielle, mais en même temps, les chamelles taries, les jeunes encore improductifs, nomadisent à des centaines de kilomètres de là dans les dunes de Chinguetti ou d'ailleurs comme si « élevage moderne » et « élevage traditionnel » se partageaient désormais l'espace au sein d'un même troupeau et donc d'une même famille d'éleveurs. En Arabie saoudite, les bédouins sédentarisés qui apprécient le confort des maisons climatisées, aiment à conserver un troupeau périurbain, sis aux franges du désert, à côté duquel ils ont planté la tente traditionnelle où ils aiment à passer le week-end en famille, buvant le lait frais de leurs animaux. Certains transhument encore sous la garde d'un berger salarié. Ainsi cohabitent encore modernité et tradition.

Gageons que la bosse ne quittera jamais complètement la dune.
\end{abstract}




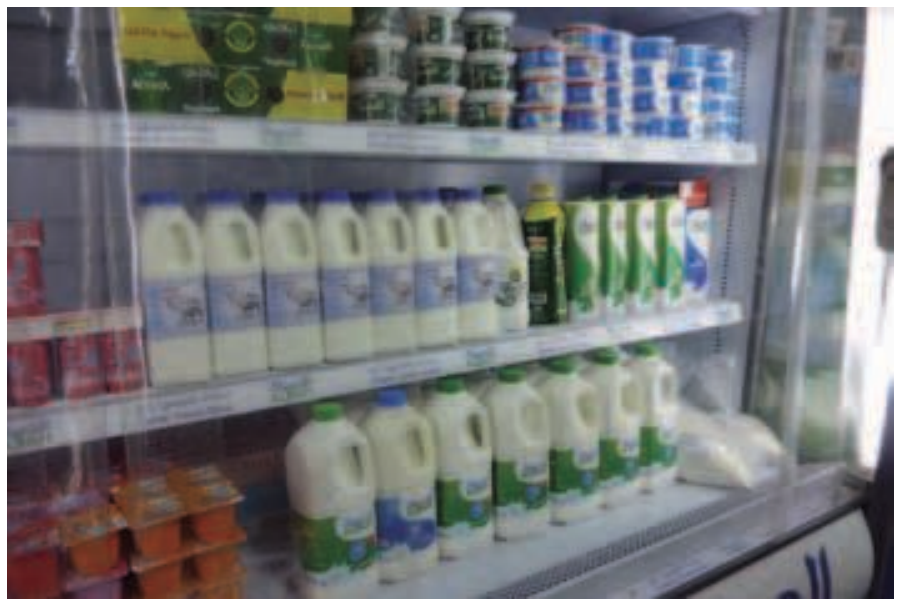

Linéaire de mini-market pour la vente de lait de chamelle pasteurisé

en bouteille plastique (Arabie Saoudite, 2011)

Les produits du dromadaire entrent ainsi dans les circuits marchands. 


\section{NOTES}

Photo d'ouverture : Dromadaires Majaheem dans les dunes du désert de Nafud en Arabie Saoudite (cl. B. Faye, 2012). Toutes les illustrations sont de l'auteur sauf mention contraire.

1. Bien que le terme générique « chameau » inclue le dromadaire (à une bosse) et le Bactriane (à deux bosses), il sera fait allusion dans ce texte essentiellement au dromadaire, le chameau de Bactriane occupant les déserts froids, plus steppiques que réellement désertiques, même si le sable n'y est pas absent.
2. Terme utilisé dans le Sahara pour parler des résurgences de nappe phréatique entre les rochers.

\section{RÉFERENCES}

Arnautovic, I. 1997 Some contributions to the study of structural adaptation of camels to the desert life, Journal of Camel Research and Practice 4 (2) : 287-293.

Bengoumi, M., Faye, B. 2002 Adaptation du dromadaire à la déshydratation, Sécheresse 13 (2) : 121-129.

Chehma, A., Faye, B. \& Djebar, M. R. 2008 Productivité fourragère et capacité de charge des parcours camélins du Sahara septentrional algérien, Sécheresse 19 (2) : 115-121.

Correra, A., Faye, B. \& Kide, A. 2011 Quelle place pour le pastoralisme dans le Parc national du Banc d'Arguin (PNBA). In S. Boulay \& B. Lecoquierre (dir.) Le littoral mauritanien à l'aube du XXI' siècle : peuplement, gouvernance de la nature, dynamiques sociales et culturelles. Paris : Éditions Khartala : 217-234.

Faye, B. 1997 Guide de l'élevage du dromadaire. Libourne : Éditions Sanofi.

Faye, B., Grech, S. \& Korchani, T. 2002 Le Dromadaire, entre féralisation et intensification, Anthropozoologica 39 (2) : 7-13.

Monod, T. 1992 Du désert, Sécheresse 3(1) : 7-24.

Rutagwenda, T., Lechner-Doll, M., Schwartz, H. J. E al. 1990 Dietary preference and degradability of forage on a semi-arid thornbush savannah indigenous ruminants, camels and donkeys, Animal Feed Science and Technology 35 : 179-192.

Trabelsi, H., Chehma, A., Senoussi, A. $E$ al. 2012 The role of the camel in the preservation of the flora covered rangeland. In E. H. Johnson $\mathcal{E}$ al. (dir.) Proceedings of the 3rd ISOCARD conference, $29^{\text {th }}$ January - $1^{\text {st }}$ February 2012, Mascate (Sultanate of Oman) : 344-345.

Yagil, R. 1985 The desert camel. Comparative physiological adaptation. London : Karger publications.

\section{POUR CITER CET ARTICLE}

Faye, B. 2014 La Dune et la bosse, in S. Boulay \& M.-L. Gélard, Vivre le sable! Corps, matière et sociétés, Techniques \& Culture $61: 60-75$. 


\section{RÉSUMÉ}

La dune et la bosse. L'image du dromadaire est indissolublement liée à celle du désert, écosystème marqué par son aridité et où se marient la pierre et le sable. Et la bosse du dromadaire n'est pas sans rappeler la rondeur des dunes. Toute l'anatomie et la physiologie du dromadaire sont tournées vers la tempérance face aux écarts de température, la récupération des nutriments lors de la digestion, la valorisation des faibles ressources pastorales par son métabolisme général, répondant ainsi à l'incapacité du sable à conserver l'eau, de fournir la matière organique des plantes et de tempérer le grand écart thermique du jour et de la nuit. Une tête faite pour le protéger des vents de sable, des pieds adaptés pour la marche dans les dunes, un métabolisme entièrement tourné vers l'économie et la tempérance, des crottes aux vertus germinatives, sont autant de paramètres permettant la survie en un milieu dominé par le sable et marqué par la mobilité des troupeaux. Tout au contraire, l'intensification cameline investit dans la sédentarisation, l'irrigation et les compléments alimentaires. Mais à ce jeu, le dromadaire perd de sa capacité à valoriser les zones les plus marginales du monde, et on peut s'interroger sur l'évolution de la qualité de ses produits tels que lait et viande dans un contexte où s'appauvrit la diversité des plantes consommées. L'avenir se situe sans doute dans une dualité impliquant sédentarisation des femelles laitières et des animaux engraissés et mobilité de la partie non-productive du troupeau comme cela s'observe de plus en plus aux marges du désert.

\section{MOTS CLÉS}

Dromadaire, adaptation, désert, mobilité, sédentarisation, intensification

\section{ABSTRACT}

Dune and hump. The image of the dromedary camel is indissolubly related to that of the desert, ecosystem marked by its aridity and where stones and sand are married. And the hump of the dromedary is not without pointing out the roundness of the dunes. All the anatomy and the physiology of the dromedary are turned towards temperance vis-a-vis the temperature variations, the recovery of the nutrients during digestion, the valorization of the scanty pastoral resources by its general metabolism, thus answering to the impossibility of sand to preserve water, provide the organic matter of the plants and moderate the great thermal variation between day and night. A head made to protect it from the sand winds, feet adapted for walk in the dunes, a metabolism entirely turned towards the economy and temperance, droppings with germinal virtues, are as many parameters allowing survival in a milieu dominated by the sand and marked by the mobility of the herds. At reverse, the camel intensification invests in sedentarisation, irrigation and food complements. But in this situation, the dromedary loses its capacity to develop the most marginal zones of the world, and the quality of his products such as milk and meat could be changed in a context where the diversity of the consumed plants is impoverished. The future is undoubtedly in a duality implying sedentarisation of milk producers or fattened animals and mobility of the non-productive part of the herd as that is observed more and more in the desert margins.

\section{KEYWORDS}

Dromedary, adaptation, desert, mobility, sedentarisation, intensification 